\title{
Golgi requires a new casting in the screenplay of mucopolysaccharidosis II cytopathology
}

\author{
Kinga Molnár ${ }^{1}\left[\right.$ J Julianna Kobolák ${ }^{2}\left(\mathbb{D} \cdot\right.$ András Dinnyés ${ }^{3,4}$
}

Received: 23 August 2021 / Accepted: 12 November 2021 / Published online: 27 November 2021

(c) The Author(s) 2021

\begin{abstract}
Lysosome (L), a hydrolytic compartment of the endo-lysosomal system (ELS), plays a central role in the metabolic regulation of eukaryotic cells. Furthermore, it has a central role in the cytopathology of several diseases, primarily in lysosomal storage diseases (LSDs). Mucopolysaccharidosis II (MPS II, Hunter disease) is a rare LSD caused by idunorate-2-sulphatase (IDS) enzyme deficiency. To provide a new platform for drug development and clarifying the background of the clinically observed cytopathology, we established a human in vitro model, which recapitulates all cellular hallmarks of the disease. Some of our results query the traditional concept by which the storage vacuoles originate from the endosomal system and suggest a new concept, in which endoplasmic reticulum-Golgi intermediate compartment (ERGIC) and RAB2/LAMP positive Golgi (G) vesicles play an initiative role in the vesicle formation. In this hypothesis, Golgi is not only an indirectly affected organelle but enforced to be the main support of vacuole formation. The purposes of this minireview are to give a simple guide for understanding the main relationships in ELS, to present the storage vacuoles and their relation to ELS compartments, to recommend an alternative model for vacuole formation, and to place the Golgi in spotlight of MPS II cytopathology.
\end{abstract}

Keywords Endo-lysosomal system · Lysosomal storage disease $\cdot$ ERGIC $\cdot$ Golgi $\cdot$ RAB2 $\cdot$ LAMP

\section{Introduction}

Lysosome, as a membrane-bound organelle, in collaboration with the endosomal system (ES), is a component of the ELS. Due to its membrane composition and hydrolytic enzyme content, the lysosome is able to degrade extra- and intracellular proteins and lipids. Besides being the site of degradation, its cytoplasmic membrane surface served as a hub for regulation of anabolic (secretory-biosynthetic) and catabolic (hetero- and autophagic) pathways. In addition,

Kinga Molnár

kinga.molnar@ttk.elte.hu

1 Department of Anatomy, Cell and Developmental Biology, ELTE, Eötvös Loránd University, Pázmány Péter stny. 1/C, Budapest 1117, Hungary

2 Department of Aquaculture, Institute of Aquaculture and Environmental Safety, Hungarian University of Agriculture and Life Sciences, Gödöllő 2100, Hungary

3 BioTalentum Ltd, Gödöllő 2100, Hungary

4 Department of Physiology and Animal Health, Institute of Physiology and Animal Health, Hungarian University of Agriculture and Life Sciences, Gödöllő 2100, Hungary lysosomes seem to be key organelles in the pathogenesis of several disorders such as storage disorders (Platt et al. 2012) and neurodegenerative diseases (Nixon \& Cataldo, 2006, Laszlo et al. 1992). Lysosome, as autonomous organelle, was discovered by Christian de Duve and colleagues during rat liver tissue fractionation experiments in 1955. The name of the lysosome (lysis - digestive or loose, soma - body) emphasizes the presence of hydrolytic enzymes being active in an acidic environment (Appelmans et al. 1995). Besides the originally discovered ribonuclease, deoxyribonuclease, cathepsin, and acid phosphatase, until now more than 60 lysosomal enzymes have been described (Rigon et al. 2021). The $\mathrm{pH}$-dependent enzyme activities guarantee the assignment of catabolized reactions to the well-defined compartment of ELS. Lysosomal acidic $\mathrm{pH}$ is provided by vacuolar proton-translocating ATPases (V-ATPases, de Araujo et al. 2020).

Extra- or intracellular origin of lysosomal content is defined by mTORC1 (mammalian target of rapamycin complex 1), the master regulator protein of energy metabolism. Under physiological conditions, when nutrients are ready to uptake and amino acids are available due to lysosomal hydrolysis, subunits of mTORC1 (mTOR, Raptor, Pras40, 
mLST8; Yip et al. 2010) are recruited to the lysosomal membrane. Active mTORC1 limits the extend of the lysosomal compartment in two ways. On the one hand, mTORC1 phosphorylates and hereby inhibits the transcription factor EB (TFEB) responsible for lysosomal biogenesis via stimulating transcription of several lysosomal genes. On the other hand, mTORC1 limits the substrates targeted to lysosomes by inhibiting autophagy. Amino acids liberated by lysosomes influence mTORC1 activity. Under starvation conditions mTORC1 complex dissociates, unphosphorylated TFEB translocates from the cytoplasm to the nucleus to initiate lysosomal biogenesis, and autophagy also activated (Puertollano, 2014). Extracellular substrates are transported to lysosomes by the ES, while intracellular substrates by the autophagy.

Lysosomal storage disorders are rare diseases with multiorgan involvement, in which undigested material accumulates in ELS compartments due to missing or impaired lysosomal functions. Accumulated organelles, named storage vacuoles (vesicles), have characteristic morphology with diagnostic relevance. The high diversity and complexity of LSDs clearly indicate the central role of lysosomal functions in eukaryotic cell metabolism and raise demand for disease, or even patient-specific therapies.

This minireview provides a brief overview of ELS pathways, gives an insight into the nomenclature of lysosomal organelles, and introduces the main cytopathological hallmarks of MPS II. Finally, it proposes a refined in vitro model for the development of MPS II cytopathology, in which Golgi and ERGIC, as the anteroom of Golgi, play a central role.

\section{Membrane traffic routes to lysosomes}

In eukaryotic cells, endocytosed cargoes (nutrients, receptor-ligand and plasma membrane complexes, extracellular matrix components, lipids, cell-debris, pathogens) are internalized by invagination of the plasma membrane (PM) and formation of vesicles through membrane fission. Several endocytic pathways were identified and classified by their morphology and molecular features (see for details in Doherty \& McMahon, 2009).

The classical pathway is composed of a recycling circuit back to the PM and a feeder pathway for delivering selected membrane components and intake from recycled circuit to the degradative system (Huotari \& Helenius, 2011). Endocytic material is received by common early endosomes (EEs) located just beneath the PM. Individual EEs are able to undergo homotypic fusion and form a tubulo-cisternal network that exhibits a highly reticular morphology in some cell types. This pleiomorphic organelle is the recycling endosome (RE). EE sub-regions and $\mathrm{RE}$ are responsible for sorting membrane components and proteins accordingly its destination back to the cell surface or towards the feeder pathway for degradation (Scott et al. 2014). During the latter process, the membrane of EE vesicular region expands and becomes suitable for cargo transport because of its high surface area-volume ratio. This domain produces intraluminal vesicles (ILV) and becomes multivesicular endosome, which detaches from EE as free endosomal carrier vesicle or multivesicular body (ECV/MVB, Scott et al. 2014, Huotari $\&$ Helenius, 2011). After forming, the spherical ECV/MVB is delivered towards the central cytoplasmic region, while its inner $\mathrm{pH}$ drops to 5.5 .

ECV/MVBs are not prone to homotypic fusion but are able to fuse with late endosomes (LEs). LE has pleiomorphic morphology with membrane-rich multilamellar and/or multivesicular content. Unlike ECV/MVB, LE has a predisposition to homotypic fusion (Scott et al. 2014). However, it has the ability to fuse with an autophagic vacuole (AP) to form a hybrid organelle amphisome (AS). Amphisome is integrated both in the autophagic and endocytic pathway; thus, it contains substrates with extra- and intracellular origin as well (Klionsky et al. 2014). LE and AS become the active degradation site of ELS due to fusing ability with hydrolytic lysosomes.

\section{Which endosome compartment can be considered as lysosome?}

The question is not unnecessary because the embarrassingly many names and synonyms that are in use for endo-lysosomal compartments are all reflecting different perspectives. Definition of lysosome must refer to its hydrolytic enzyme content synthetized by the secretory pathway (rough endoplasmic reticulum, RER, and Golgi). In the trans-Golgi network (TGN), the newly synthesized hydrolases with mannose-6-phosphate residues are packaged into small vesicles. These complete "mini organelles" were termed primary lysosomes, which is also called the true, pure, or original lysosome. The primary lysosome hypothesis focuses on the vesicular content and $\mathrm{pH}$ : primary lysosome delivers hydrolytic enzymes without substrates (Ghadially, 1988), and $\mathrm{pH}$ is above 6 . In contrast, a secondary lysosome is a substrate containing digestive organelle characterised by $\mathrm{pH}<6$. The primary lysosome can fuse with LE, (hetero) phagosome, and autophagosome (AP); thus, endolysosome (EL), phagolysosome, and auto(phago)lysosome (AL) are formed, respectively (term heterolysosome incorporates phago- and autolysosome populations; Ghadially, 1988). Organelle given by fusion of amphisome (AS) and lysosome is regarded as endolysosome.

Characteristic functions of EE (receptor-cargo dissociation), ECV/MVB, and LE/lysosome (hydrolysis) are 
governed by intraluminal $\mathrm{pH}$ (EE: $\mathrm{pH} \sim 6.5$, ECV/MVB: $\mathrm{pH}$ 5.5; Scott et al. 2014, LE: pH 6.0-4.9; Huotari \& Helenius, 2011). Only acidic pH provides the optimal environment for lysosomal hydrolases. By definition, endolysosomes and autolysosomes should be regarded as secondary lysosomes due to be the active degradation site. It should be mentioned here that hydrolytic enzymes and their substrates can get into common vesicle not only by above-mentioned fusion processes, but transient "kiss-and-run" contacts as well (Luzio et al. 2007). Through acidification, V-ATPases control not only intraluminal functions but the formation of multivesicular transport intermediate MVBs and the size of the LEs by fission and fusion events (de Araujo et al. 2020).

ELS organelles are dispersed and undergo continuous maturation and cyclic fusion and fission events. An example of the latter is the process of autophagic lysosome reformation (ALR), in which the so-called reformation tubule (tubular subdomain of autolysosome membrane; Rong et al. 2012) forms small newly formed proto-lysosomes (de Araujo et al. 2020). ALR is thought to restore the level of free, recyclable lysosomes (Chen \& Yu, 2018) because they mature into functional lysosomes (Chen Yu, 2017). Whether protolysosomes are primary lysosomes? If it is a lysosome formed for the purpose of recycling, why would it be primary? In point of function, naming inactive and active seems to be more practicable. And what about the name lysosome and what route do lysosomal enzymes and membrane components take before they form a "ready to use" organelle? Getting to know the ultrastructural events and molecular background better, terms primary and secondary lysosomes became questionable.

For clarity look at the simplest molecular approach. Molecular characterization of the endomembrane system of mammalian cells has been detailed by several comprehensive reviews elsewhere (Scott et al. 2014, WandingerNess \& Zerial, 2014, Langemeyer et al. 2018, Henne, 2016, Li et al. 2020, Mellman \& Nelson, 2008). This summary is confined to some often-used markers for immunocytochemical identification. ELS and the secretory-biosynthetic pathway are related to each other through several routes. GM130 (130 kDa cis-Golgi matrix protein or Goga2, Golgin subfamily A 2 or Golgin2A) is one of the best-studied Golgin, which mediates RER-Golgi and intra-Golgi transport. Additionally, GM130 is involved in ribbon formation: its phosphorylation (before cell division) or functional absence (e.g. in GM130 knockout mouse) causes Golgi fragmentation (Liu et al. 2017). Traffic between RER and Golgi is mediated by RAB2 (small GTPase Ras-related protein 2) as well (Lund et al. 2018), where GM130 is a subunit of RAB2 effector complex on the Golgi membrane (Liu \& Storrie, 2012). Whereas RAB2 localises on RER, ERGIC, and Golgi membranes, GM130 can be identified only in ERGIC and Golgi compartments. ERGIC is a stationary compartment for anterograde and retrograde sorting, concentration, folding, and quality control of newly synthesized proteins. The possibility of RAB2 function in retrograde vesicle transport from Golgi to ERGIC was also formulated (AppenzellerHerzog \& Hauri, 2006).

EE membrane recruits RAB5 and EEA1 (early endosome antigen 1), while the recycling route to the $\mathrm{PM}$ is mediated by RAB4 and RAB11. Characteristic RAB of ECV/MVB, $\mathrm{LE}$, and lysosome is the RAB7 protein. RAB7 positive organelles can fuse with each other. ELS compartments compose a dynamic and adaptable continuum, in which RABs and their occasional combinations give clear cues for identification of compartments, enables sorting and exact targeting of membrane components and cargo. Besides RABs, SNAREs (soluble N-ethylmaleimide-sensitive fusion protein (NSF) attachment protein receptors) and multi-subunit tethering complexes (HOPS, homotypic fusion and protein sorting, CORVET, class $\mathrm{C}$ core vacuole/endosome tethering and CHEVI, class $\mathrm{C}$ homologs in endosome-vesicle interaction) control homo- and heterotypic membrane fusion (see more details in van der Beek et al. 2019 and Jiang et al. 2014).

The lysosomal membrane can be identified by its LAMP (lysosome-associated membrane glycoprotein) positivity (Platt et al. 2012) LAMP-1 and LAMP-2, which are estimated to contribute to about $50 \%$ of all proteins of the lysosome membrane. The highly glycosylated luminal domain of LAMPs forms a glycocalyx-like layer on the inner surface of the vesicle; therefore, both LAMPs were thought to protect the lysosomal membrane against hydrolytic enzymes (Eskelinen, 2006).

Lysosomes receive substrates both via endosomal and autophagic pathways. LC3 (microtubule-associated protein 1A/1B-light chain 3) is a widely used autophagosome marker (Mizushima \& Yoshimori, 2007). It is recruited to the isolating membrane during phagophore formation and accompanies the sequestered material to the end of its degradation; therefore, LC3 is detectable on AP, AS, and AL (Tanida et al. 2004). AL formed proto-lysosomes are LAMP1 positive but LC3 negative small vesicles (de Araujo et al. 2020). Figure 1 gives an overview of the above.

The terminal event of the degradative process is forming an organelle that contains only undigested waste materials. This residual body is often mentioned as tertiary, residualor telolysosome (Lazarus et al. 1975). Telolysosomes are exocytosed or accumulated in the cell (a typical example of the latter is lipofuscin granules; Terman \& Brunk, 1998). It is worth mentioning that not only telolysosomes, but MVBs, LEs, AS, ELs, and ALs also are able to emit their content via exocytosis (Fig. 1). Lysosomal exocytosis is a non-conventional type of exocytosis that seems to be the major output pathway of lysosomes in healthy cells (Buratta et al. 2020). Deficit of this clearance mechanism 


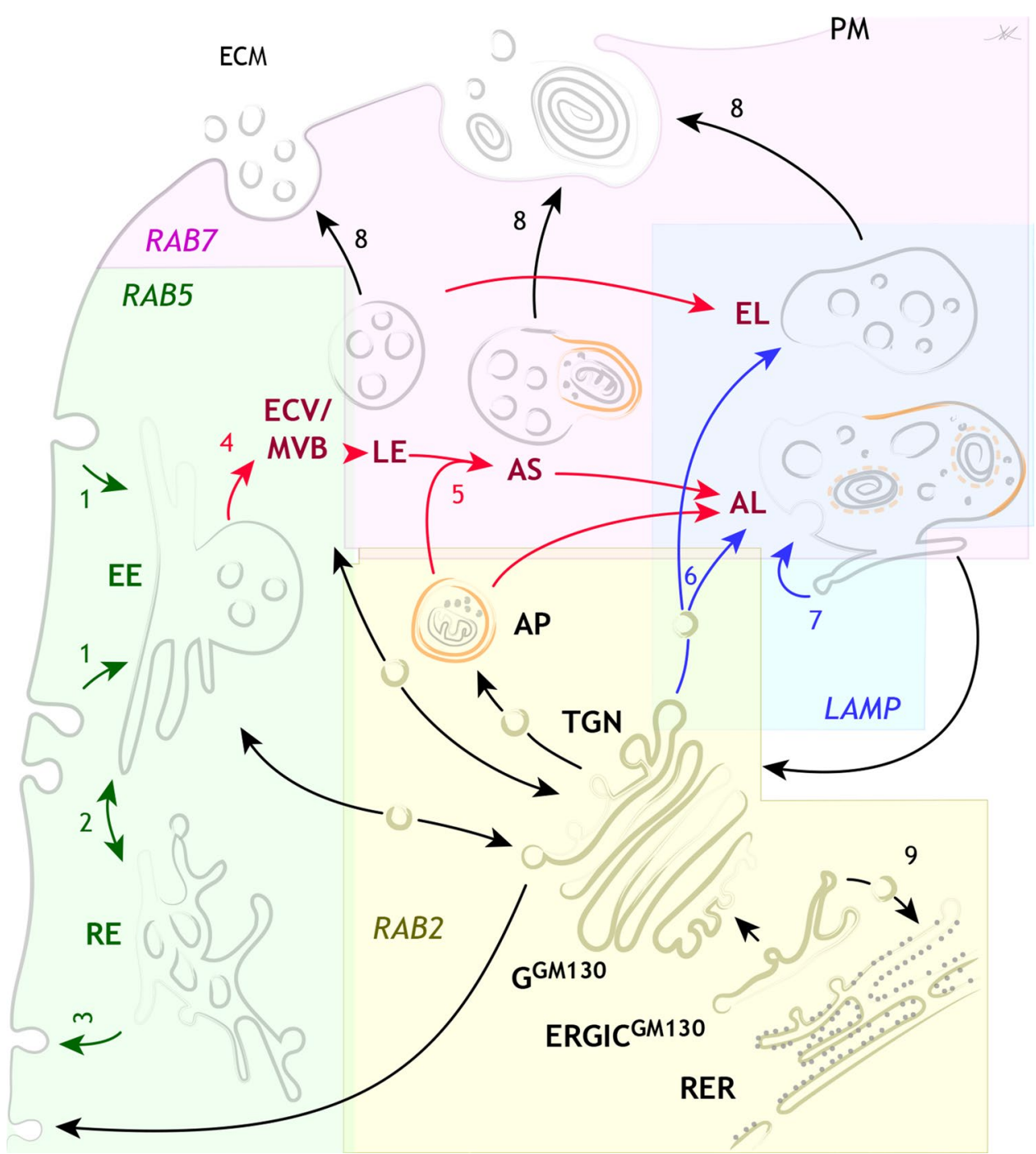

Fig. 1 Routes and identity of organelles composed of the endo-lysosomal system. Primary endocytic vesicles are received by the early endosome (EE and number 1). In the RAB5 positive recycling circuit, some cargo is sorted back to the plasma membrane (PM) via the recycling endosome (RE and numbers $2-3$ ). In the degradative pathway, the vesicular region of the early endosome forms the irrespective endosomal carrier vesicle/multivesicular body (ECV/MVB and number 4) which becomes RAB7 positive late endosome (LE) during its maturation. When the late endosome fuses with $\mathrm{LC} 3$ positive autophagic vacuole (AP), an amphisome (AS) is generated (number 5). AS and late endosomes are able to receive hydrolytic lysosomal enzymes from the trans-Golgi network (TGN). In this way (number 6), autolysosome (AL) and endolysosome (EL) are formed as a

leads to cytopathological consequences via accumulation of undigested substrates (Samie \& Xu, 2014); therefore, experimental results that lysosomal exocytosis can be stimulated transcriptionally by TFEB (Palmieri et al. 2011, Medina et al. 2011, Spampanato et al. 2013) give a promising possibility for therapies in lysosomal impairment.
LAMP positive compartment. AL is a platform of lysosome reformation (number 7), while the multivesicular body, late endosome, AS, $\mathrm{AL}$, and endolysosomes can empty their accumulated content via lysosomal exocytosis (number 8). Trans-Golgi network (TGN) has reciprocal connections with each compartment of EL system. Routes of the recycling circuit, degradative pathway, and lysosomal biogenesis are indicated by green, red, and blue arrows, respectively. LC3 positive membrane is orange. Note overlapping RAB and LAMP domains that indicate the continuum between successive components. Association of GM130 to Golgi (G) and ERGIC membranes is indicated by superscription. Possibility of RAB2 involvement in ERGIC to RER retrograde traffic is indicated by number 9

\section{Mucopolysaccharidosis II and lysosomal storage diseases}

MPS II is an LSD caused by sulfuric ester hydrolase, idunorate-2-sulphatase (IDS) enzyme deficiency. It is an $\mathrm{X}$-linked disorder with recessive heredity (manifests almost 
exclusively in males). Impaired or absent IDS activity results in disturbed glycosaminoglycan (GAG) metabolism; therefore, the main cytopathological feature of MPS II is the accumulation of undigested heparan and dermatan sulphate in storage vacuoles. Formally, LSDs can be sorted into two groups. The primary storage disorders (classic LSDs) are caused by an enzyme deficiency. In this case, the homoand heterotypic fusions of ELS organelles are not affected, but for lack of degradation, ELs and ALs accumulate, but residual bodies are not formed. In secondary storage disorders, the lysosomal hydrolytic function is intact, and fusion processes are damaged by lysosomal protein dysfunction. In primary disorders, such as MPS II, secondary substrate accumulation in the lysosomal compartment is generated by impairment of intact enzyme and permease functions by stored material. These led to disturbance of not only degradative but biosynthetic secretory pathways (RER, Golgi) through perturbed calcium homeostasis. RER and Golgi stress are common in LSDs (Platt et al. 2012). Knowing the involvement of all organelles (don't forget mitochondria) the wide range of clinical symptoms of MPS II (musculoskeletal deformities, organomegaly, disorder of sensory organs, thick skin, profound neurological dysfunctions) is not surprising. Symptoms manifest between 1,5 - 4 years of age and lead to a dramatic premature death (Platt et al. 2012; Kobolák et al. 2019).

Described more than 500 mutations of IDS make it difficult to find the common cytopathologic events. Some patients have residual IDS activity, whereas in most serious cases, enzyme activity is entirely missing. When truncated protein is expressed, it is eliminated by ER-associated degradation mechanism (ERAD) induced by misfolding and protein accumulation in RER. By inhibition of ERAD in HeLa cells, Osaki and colleagues were succeeded in partially recovering lysosomal transport and enzyme activity of IDS (Osaki et al. 2018). Standard treatments of MPS II are enzyme replacement therapy (ERT) and hematopoietic stem cell (bone marrow) transplantation. The limitation of the most successful ERT is that the recombinant human enzyme is not able to penetrate the blood-brain barrier (BBB), so ERT cannot ameliorate the central nervous system (CNS) dysfunctions.

\section{Cytopathological hallmarks of MPS II}

We established and defined (Kobolák et al. 2019) a human in vitro MPS II model based on induced pluripotent stem cell (iPSC) technique to study MPS II cytopathology, thus providing a human-relevant translational platform for the development of new therapies. In this model, MPS II patient-derived blood cells (Varga et al. 2016a, Varga et al. 2016b, Varga et al. 2016c, Varga et al. 2016d) served as a source of iPSC establishment, and then, neural cells (neural progenitor cells, NPC), and terminally differentiated 2D and $3 \mathrm{D}$ cultures were produced. The appearance of storage vacuoles as the cytopathologic hallmarks of the disease was detected in every MPS II affected cell line. By its ultrastructure, storage vacuoles can be sorted into four groups (Parkinson-Lawrence et al. 2010, Lemmonier et al. 2011). Primary vesicles (Pr) contain exclusively or predominantly floccular or fibrillar-granular material. Globular (Glo) type and multilamellar (ML) type enclose intraluminal vesicles or membrane whirls, respectively. Additionally, sequestered (Sq) vacuoles contain incorporated cytoplasmic remnants. Glo, ML, and Sq are considered as matured types (Fig. 2). All these vesicle types were detected in the in vitro (Lemmonier et al. 2011) and in vivo (Vitry et al. 2010) models of MPS III (San Filippo disease), as well.

Immunocytochemical labelling is a conventional technique to identify ELR components, which makes it a useful method to clarify the relationship of storage vacuoles and lysosomal organelles. In our study, RAB5 and EEA1 as EE marker, RAB7 as LE marker, Cathepsin D (CATD), and LAMPs as lysosomal markers were studied. On the ultrastructural level, some Glo, ML, and Sq vacuoles proved to belong to the LE compartment: not all but many of them showed RAB7 and CATD immunopositivity. The appearance of early endosomal markers was documented only inside of them. However, primary vesicles showed neither RAB5, nor RAB7/CATD immunopositivity (Kobolák et al. 2019).

These results raised the question of whether primary vesicles are part of the endosomal system? To find the answer, we incubated control and disease-affected neuronal NPC 3D cultures with TMA (11-Mercaptoundecyl-N,N,N-trimethylammonium bromide)-coated $5 \mathrm{~nm}$ gold particles (Peter et al. 2018, Kobolák et. al. 2019). After treatment, colloid was identified on the cell surface and Glo, ML, and Sq vacuoles but not in Pr vesicles (Fig. 2).

This observation suggested the hypothesis that the original content of storage vacuoles thus the material of Pr vesicles does not originate from the extracellular matrix $(\mathrm{ECM})$. The endosomal system independent origin of primary storage vacuoles was suggested previously by Vitry and colleagues after identification of GM130 and RAB7 double-positive storage vesicles in cortical neurons of MPS IIIB mouse model (Vitry et al. 2010). Short Golgi stacks and cistern deformation were documented in our iPSC-derived cultures as well, and therefore, we examined Golgi-associated GM130 and RAB2 distribution in MPS II neuronal cells. (All methods were described earlier (Kobolák et al. 2019), except 1:25 dilution of anti-GM130 antibody (BD Biosciences, 610,823) for ultrastructural detection.) After immunogold labelling, gold particles were observed on Golgi cisterns and neighbouring ERGIC 

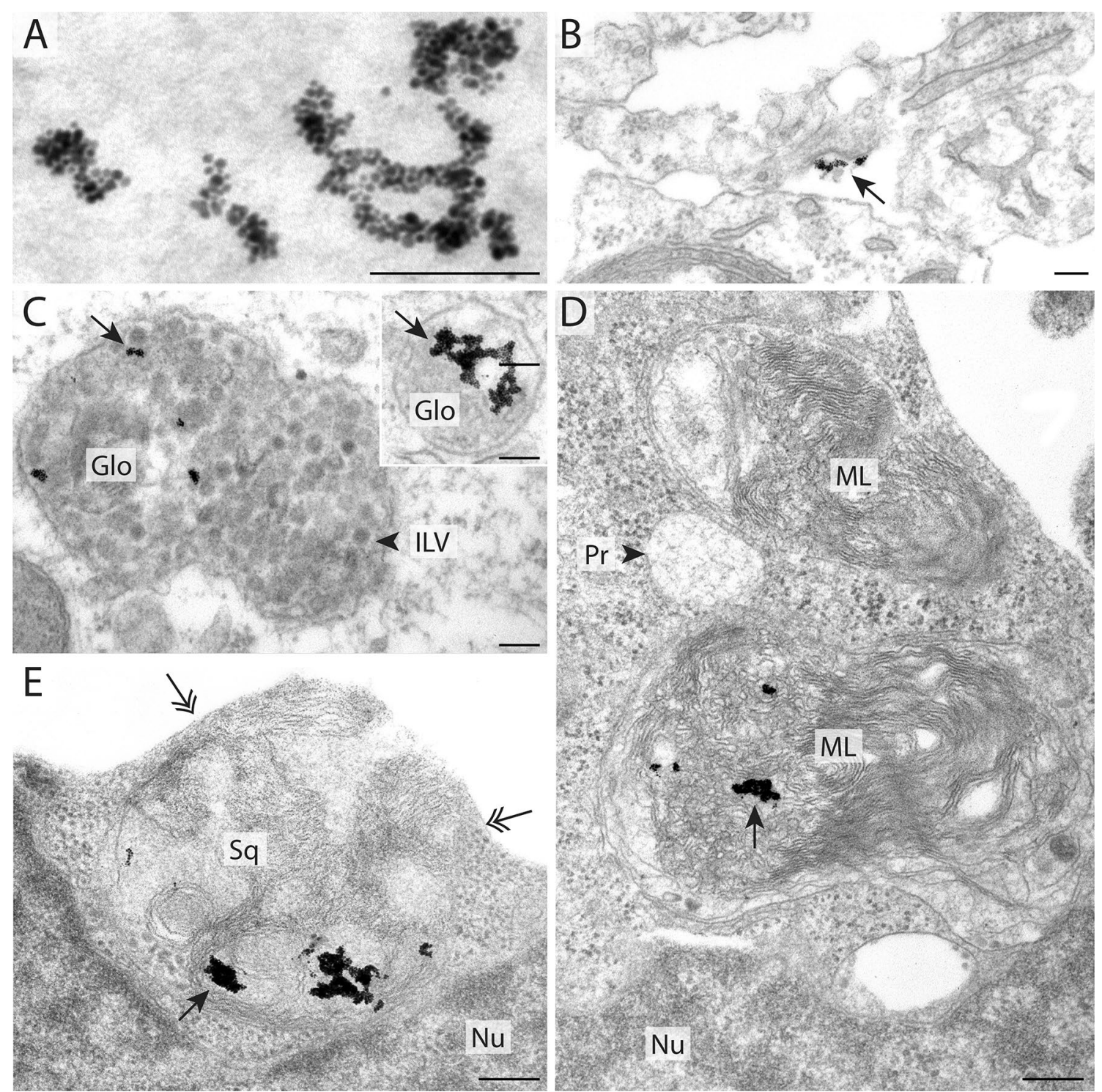

Fig. 2 Route of endocytosed TMA-coated gold particles in MPS II NPC cells. TMA colloid is indicated by a black arrow. a Shape and distribution of endocytosed TMA functionalised gold particles on high magnification. b Colloid on the cell surface. c Nanogold particles in globular type storage vacuoles (Glo). d Colloid enclosed in a multilamellar storage vacuole (ML). e Nanogold particles in sequestered type storage vacuole $(\mathrm{Sq})$ in the very moment of lysoso-

tubules and vesicles in the case of both Golgi markers, but never in the case of ER stress markers Calreticulin and GRP78 (78 kDa glucose-regulated protein). GM130 and RAB2 positive vesicles seemed to be able to fuse with storage vacuoles (Fig. 3).

In 2017, Lôrincz and colleagues showed that RAB2 promotes endocytic and autophagic lysosomal degradation in

mal exocytosis (double-headed arrows show the site of invagination of plasma membrane; Glo - globular type storage vacuole, ILV intraluminal vesicle, ML - multilamellar type of storage vacuole, $\mathrm{Nu}$ - nucleus, $\mathrm{Pr}$ - primary storage vesicle with floccular content, $\mathrm{Sq}$ - sequestered type storage vacuole; 5 min treatment: A, D, E; 10 min treatment: B, C; Scale bars: A, B, C: $100 \mathrm{~nm}$; D, E: $200 \mathrm{~nm}$ )

Drosophila and human breast cancer cells. They suggest a model in which RAB2 on Golgi-derived vesicles and RAB7 on LE, AL, and lysosome may bind indirectly via HOPS complex (Lôrincz et al. 2017). We cannot rule out the possibility of similar events in MPS II affected neuronal cells because of the appearance of Golgi markers and RAB7 on some storage vacuoles. 


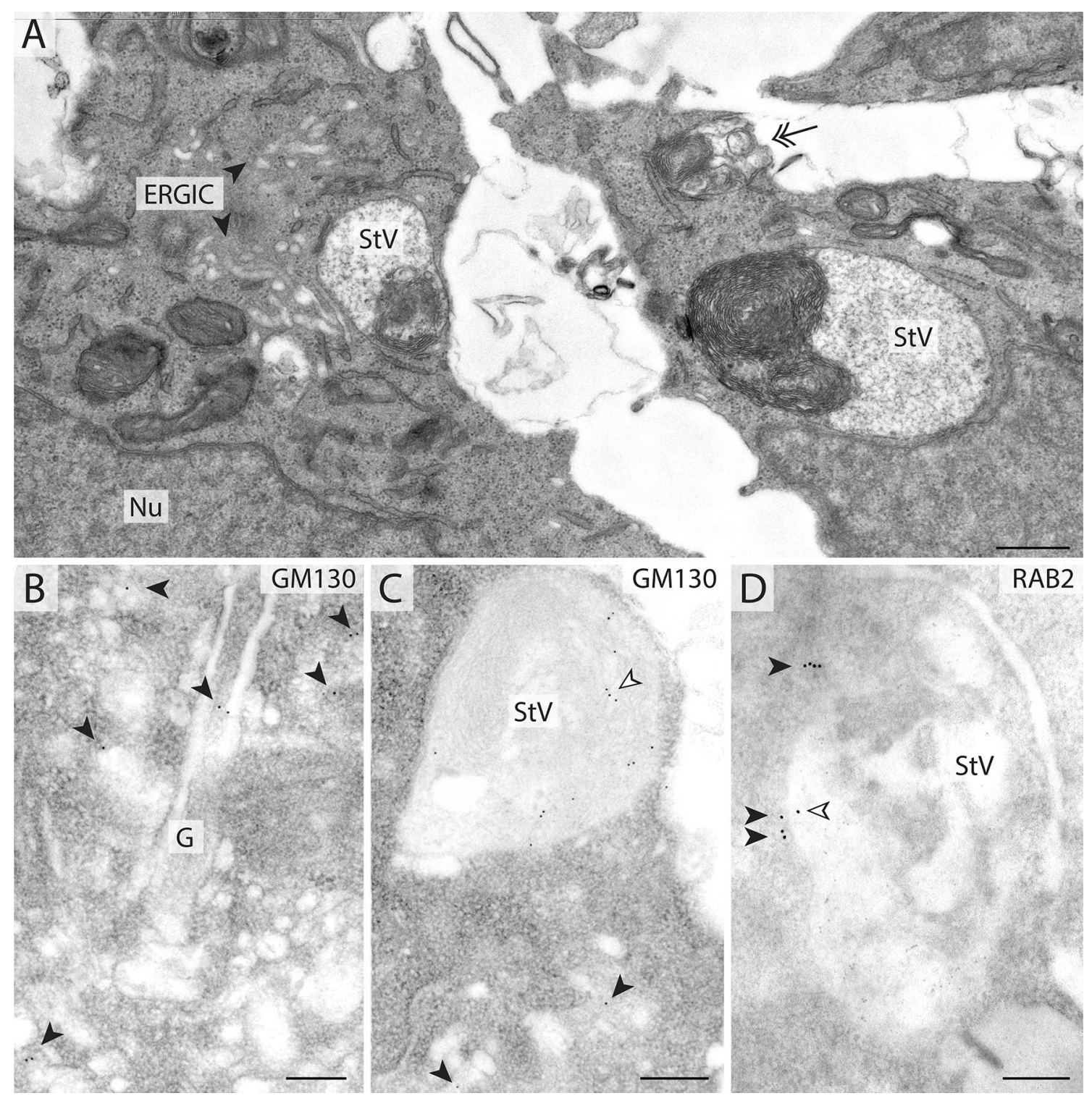

Fig. 3 ERGIC morphology and Golgi markers in storage vacuoles of MPS II NPC cells. a Extended and dilated ERGIC tubules, storage vacuoles ( $\mathrm{StV}$ ), and lysosomal exocytosis (double-headed arrow) in two adjacent cells. b-d $10 \mathrm{~nm}$ gold particles refer to Golgi marker GM130 and RAB2 localization in Golgi cistern (G) and vesicles are indicated by black arrowheads, while Golgi marker localization

\section{What is the origin of primary storage vesicles?}

Routes of lysosomal biogenesis are not as simple as we earlier thought. The original primary lysosomal hypothesis said that newly formed lysosomes originate from TGN via budding as complete, miniature organelles.

Biosynthetic delivery of lysosomal proteins follows two routes. Soluble proteins are transported in clathrin-coated vesicles from TGN to PM, EE, MVB, or LE. If primary storage vesicles would be the same as one of the latter, its in storage vacuoles $(\mathrm{StV})$ is pointed by white arrowheads. b GM130 localization in Golgi cistern and surrounding vesicles. c GM130 in storage vacuole and ERGIC. d RAB2 positive membrane of storage vacuole and tiny vesicles (scale bars: A: $500 \mathrm{~nm}$; B, C: $50 \mathrm{~nm}$; D: $100 \mathrm{~nm})$

limiting membrane should be RAB5 or RAB7 positive. However, such primary vesicles were not observed in MPS II cells. The second route is followed by lysosomal membranebound proteins, like LAMPs. It is direct way from TGN which is mediated by non-clathrin coated, biosynthetic/ lysosomal membrane protein (LMP) carrier (Lund et al. 2018). LMP carriers are expected to be RAB2 and LAMP positive but RAB7 negative vesicles. Except that LAMP carriers have no clathrin coat, this route is most in line with the original primary lysosome hypothesis. 
In their publication, Lund reported that in Drosophila garland cells, i) RAB2 is required for the delivery of LMP carriers to endosomes, in the absence of RAB2, ii) LMP carriers accumulate and fuse to each other, and iii) form LAMP positive non-degradative storage lysosomes (Lund et al. 2018). Despite lacking ultrastructural characterization of fruit fly LMP carriers and functional examination of RAB2 compartment in iPSC-derived MPS II cell lines, these observations seem to be consistent with our results: enlargement of the LAMP positive compartment was one of the most prominent features of MPS II affected terminally differentiated neuronal cells (Kobolák et al. 2019). We identified extended ERGIC both in NPC and TD cultures (Kobolák et al. 2019). The most notable observation was the physical contact of primary storage vesicles and cistern-like spaces (Fig. 4). However, we detected continuity between a wide, ribosome-free RER cistern and storage vesicle (Kobolák et al. 2019), which refers to the perturbation of vesicle transport between RER and Golgi.

Based on all together, we can assume that RAB2/GM130 vesicles and RAB2/LAMP positive LMP carriers seem to be the best candidates as a source of primary storage vesicles in MPS II. Consequently, these ERGIC and Golgi vesicles deliver floccular content and membranes into the matured storage vacuoles via fusion with RAB7 negative endolysosome/autolysosome-derived organelles. Impairment of RAB2 functions also can be assumed in MPS II. It may confuse the fusion of not only LPM carriers to LE compartments but ERGIC-derived vesicles to RER cisterns. Besides RABs, bidirectional sorting of ERGIC may be mediated by the members of ARF (ADP ribosylation factor) small GTPase family. After knocking down of ARFs in different combinations, inhibition of vesicle budding, enlargement of the ERGIC compartment, and blocking of anterograde and

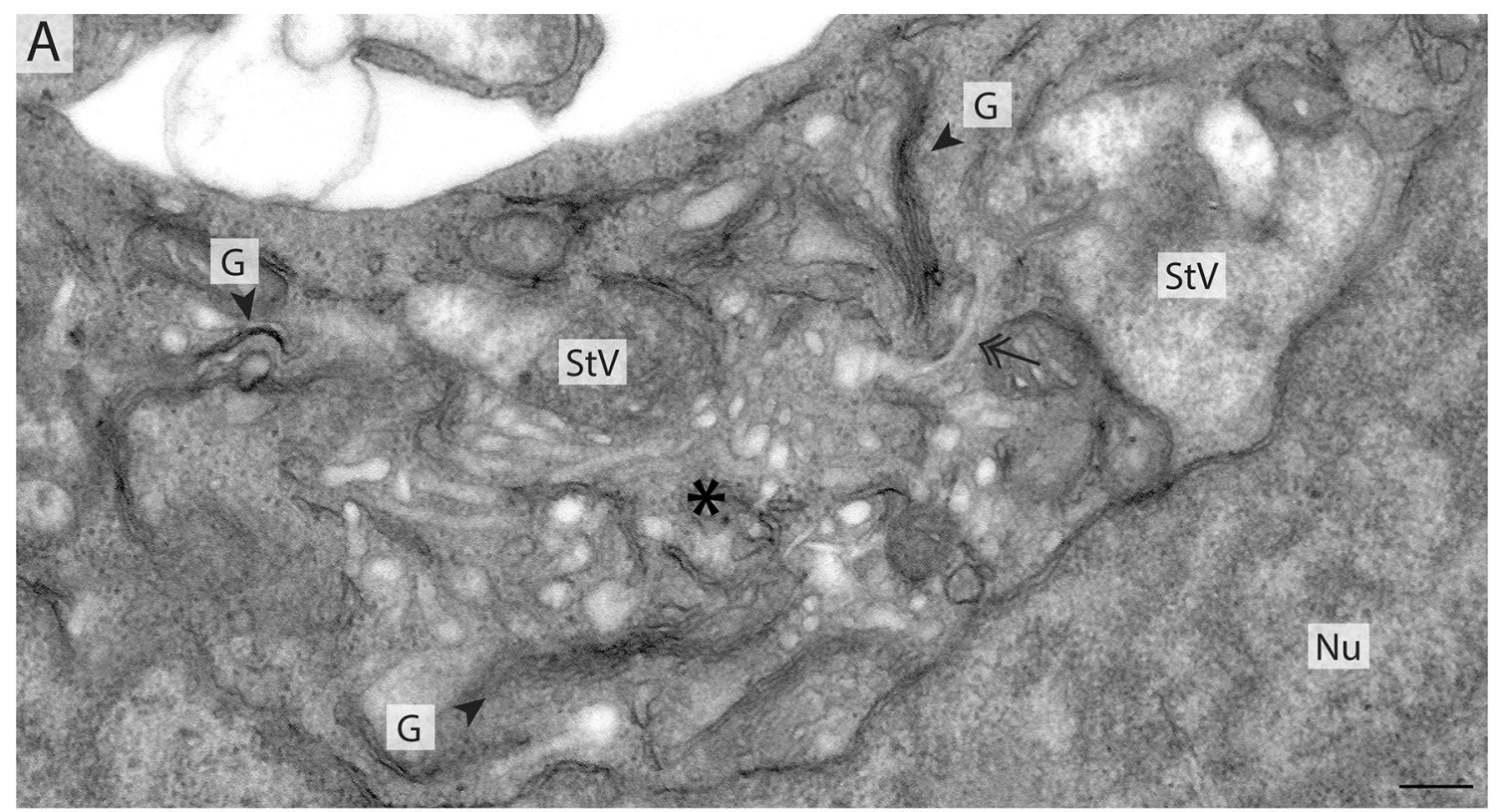

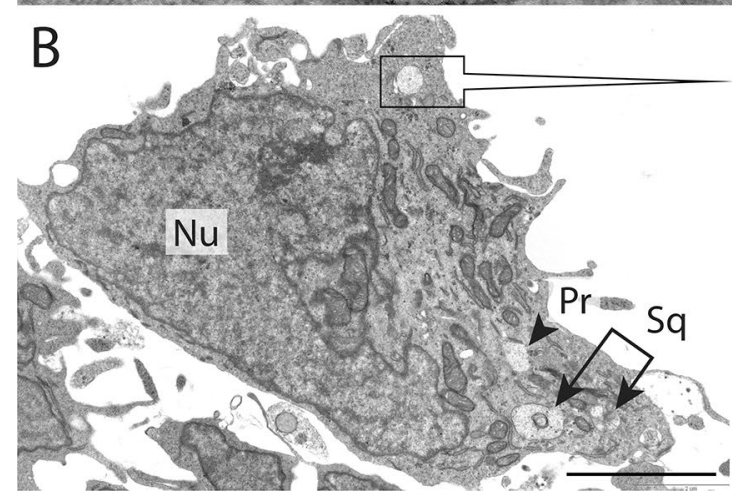

Fig. 4 Golgi and ERGIC deformation in MPS II NPC cells. a Extended and dilated ERGIC (asterisk) with a long tubule stretching towards a storage vesicle (double-headed arrow) between deformed Golgi stacks $(\mathrm{G})$. b Overview of an NPC cell with primary (Pr) and

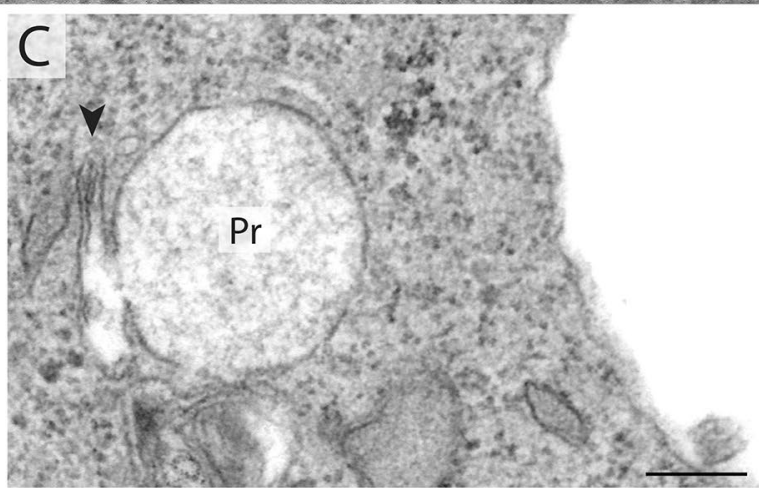

sequestered $(\mathrm{Sq})$ storages vesicles. Framed part is shown by panel C. c A primary storage vesicle with cistern-like contact (arrowhead; scale bars: A, C: $200 \mathrm{~nm}, \mathrm{~B}: 2 \mu \mathrm{m}$ ) 
retrograde transport were observed (Appenzeller-Herzog \& Hauri, 2006). Almost the entire quantity of synthetized proteins in RER passes through the ERGIC, and this amount is increased by TFEB induced lysosomal biogenesis in MPS II. We complemented our earlier model and raise the possibility that the floccular material of storage vesicles origin from the ERGIC (Fig. 5).

Autophagosome accumulation is thought to be common in many storage diseases. Elevated LC3-I expression, induced autophagosome formation but impaired LC3 maturation was detected in our iPSC-derived TD neuronal cultures. However, NPC cells were successful in problem management by reducing their GAG uptake and stimulating lysosomal exocytosis. In contrast, TD cultures are prone to accumulate GAG and enlarge the LAMP compartment. Expansion of LAMP compartment was not accompanied by RAB 7 compartment (Kobolák et al. 2019), as if LAMP positive-RAB7 negative organelles got outside the ELS (Fig. 5). In accordance with the literature (Rama Rao \& Kielian, 2016), we found that astrocytes are responsible for elevated GAG accumulation of neural tissue. Massive accumulation leads to incremented Caspase $3 / 7$ activity and vulnerability for apoptotic cell death (Kobolák et al. 2019).

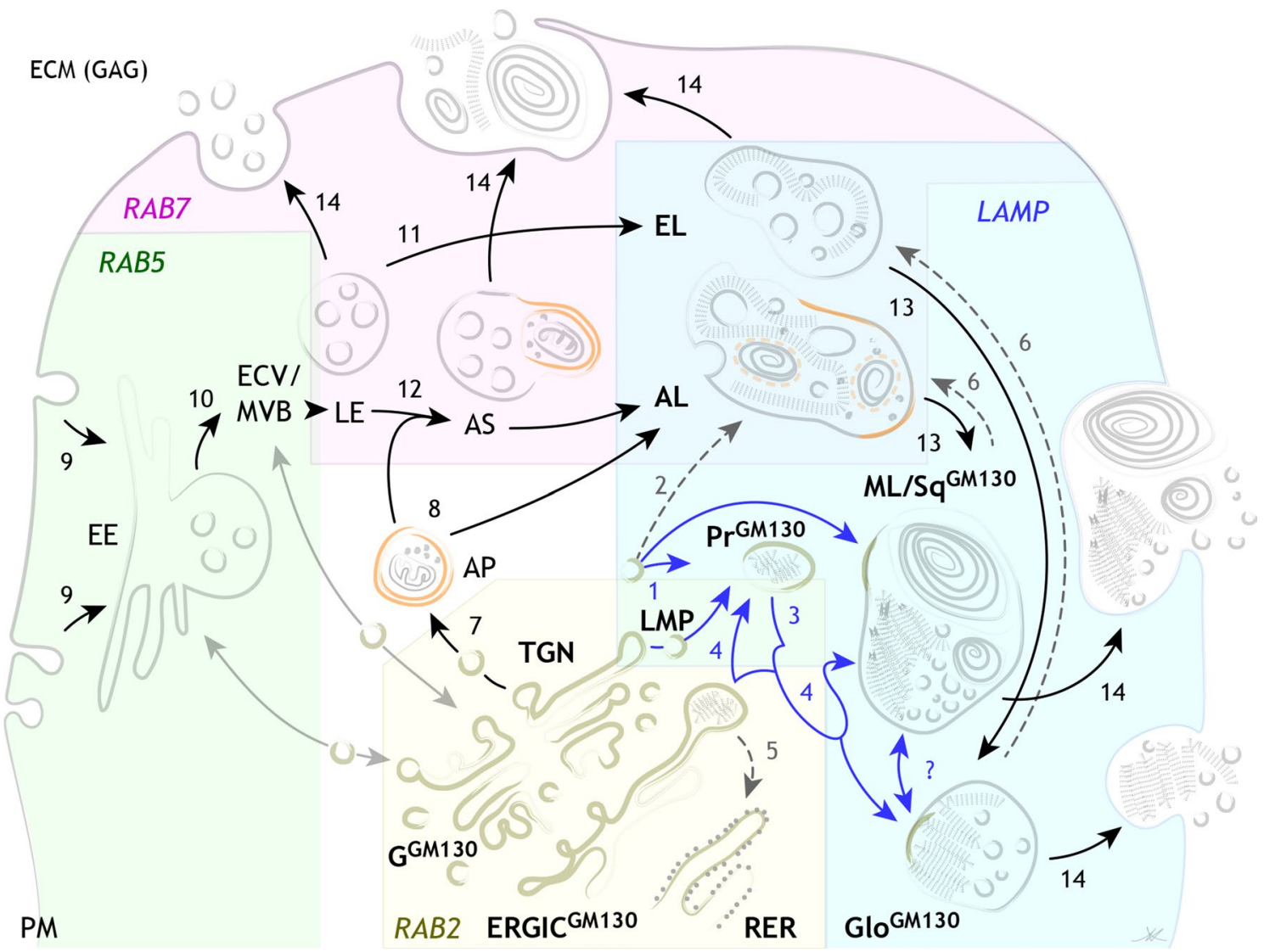

Fig. 5 Putative model for the generation of storage vacuoles in MPS II neuronal cells. Primary storage vesicles (Pr) are formed by the fusion of Golgi-derived LAMP positive-RAB7 negative lysosomal membrane protein (LMP) carrier vesicles (number 1) that are rather prone to fuse to each other than to RAB7 positive endolysosomes and autolysosomes (number 2). Because fusion with RAB7 compartment is impaired, primary storage vesicles transport LAMP positive membranes to RAB7 negative organelles (3) origin from endolysosomes and autolysosomes (number 13). In this way, matured storage vacuoles (Glo - globular type, ML - multilamellar type, Sq - sequestered type) are produced. Putative ERGIC-derived vesicles also can fuse with storage vesicles (number 4) but are not able to recycle back to the RER (number 5, dashed line). The ability of matured storage vacuoles to re-enter the RAB7 compartment is also questionable (number 6, dashed line). Extracellular matrix components (ECM) such as GAGs and nanogold colloids achieve the matured storage vacuoles via the endocytic pathway (numbers 9-13). Autophagy (numbers $7-8,12$ ) terminates without degradation and increases the number of storage vacuoles (number 13). At the tissue level, TFEB induced lysosomal exocytosis caused the accumulation of undigested materials in ECM (number 14). Note the fragmented Golgi (G), the large dilatations at the end of ERGIC tubules filled with material of primary storage vesicle and shrunk (pale yellow) RAB2 compartment. Dashed lines indicate impaired routes 


\section{Golgi is a key player in MPS II cytopathology}

Our hypothesis that ERGIC and Golgi-derived LMP carriers are responsible for the formation of primary storage vesicles, placing Golgi in the focus of MPS II cytopathology. RER and Golgi stress-induced cytopathology are thought to be a consequence of lysosomal dysfunction generated by IDS mutations, but events behind the scenes are far from understood.

Whether truncated or missed IDS per se, is able to influence ERGIC and Golgi morphology? By a high throughput interactomics approach, Huttlin and colleagues raised the possibility of the existence of physical contact between IDS and ERGIC2 (Huttlin et al. 2015). ERGIC2 (endoplasmic reticulum-Golgi intermediate compartment protein 2, Erv41, Ptx1) is a transmembrane protein with localization in RER to Golgi route (RER, ERGIC, Golgi) and predicted function in vesicular transport (Gaudet et al. 2011). ERGIC2 and GM130 colocalise in ERGIC tubules and Golgi cisterns, and Ergic2 has the capability and possibility to bind a Golgin protein GM130 (Go et al. 2021), which has a proven role in ER to Golgi transport and regulation Golgi morphology (Appenzeller-Herzog \& Hauri, 2006, Liu et al. 2017). We know multiple transcript variants (splice IDS mutants are a considerable source of MPS II pathophysiology) and more than 500 mutations of IDS (Alves et al. 2006). Can these variants be translated into peptides and proteins or exist as mRNAs? What are their interactions and what is their lifetime in the secretory-biosynthetic pathway? Unknowing the answers we cannot exclude the possibility that any of them are able to influence the ERGIC and Golgi functions not only indirectly but in a direct way as well. However, a recently described genetically modified Drosophila MPS II model in which IDS activity was impaired (but not abolished) by neural tissue-specific mRNA interference did not show GAG accumulation and LAMP compartment enlargement (Rigon et al. 2020). This result suggests that a more complex approach in which all hypothetic primary and secondary consequences of IDS malfunctions are examined should be considered.

\section{Conclusion}

Features and consequences of Golgi stress are seldom addressed by LSD reviews. Our data suggest that assumable malfunctions of RAB2/GM130 compartment, the ratio of RAB2/RAB7, RAB2/LAMP compartments, involvement of RAB1 functions in ERGIG to Golgi anterograde transport, fission defects, and fusion ability of ERGIC and
Golgi-derived vesicles also should be considered and studied further. Considering the complexity of MPS II pathology, IDS predisposition to generate splice variants, and the predicted pleiotropic effect of IDS on biosynthetic pathways, worthy of applying the less complex, in vitro model system to investigate the threads of disease. In exploring the key players of cytopathology, patient-derived human in vitro models may be more successful than in vivo animal models. Overall, experimental data discussed in the present study indicate that improvement of Golgi functions in MPS II may be a good target towards improvements of clinical pathologies of this disease in the future.

Acknowledgements The authors thank Dr. Lajos László (Dept. Anatomy, Cell and Developmental Biology, ELTE, Eötvös Loránd University, Budapest) for consultations and review of the manuscript and Eszter Varga (BioTalentum Ltd, Gödöllő) for the establishment of the MPS II-patient iPSC lines.

Author Contributions KM wrote the manuscript and performed the electron microscopy analysis. JK was involved in the implementation of the experiments, and interpretation of the results and revised the manuscript. AD provided the MPS II iPSC line, the differentiated cells and revised the manuscript.

Funding Open access funding provided by Eötvös Loránd University. This work was supported by grants from European Union's FP7-PEOPLE programme (Anistem, PIAPP-GA-2011-286264; STEMMAD, PIAPP-GA-2012-324451). FP7 People: Marie-Curie Actions, PIAPPGA-2011-286264, András Dinnyés, PIAPP-GA-2012-324451, András Dinnyés

\section{Declarations}

Conflict of interest The authors declare no competing interests.

Ethical Statement Not applicable.

Open Access This article is licensed under a Creative Commons Attribution 4.0 International License, which permits use, sharing, adaptation, distribution and reproduction in any medium or format, as long as you give appropriate credit to the original author(s) and the source, provide a link to the Creative Commons licence, and indicate if changes were made. The images or other third party material in this article are included in the article's Creative Commons licence, unless indicated otherwise in a credit line to the material. If material is not included in the article's Creative Commons licence and your intended use is not permitted by statutory regulation or exceeds the permitted use, you will need to obtain permission directly from the copyright holder. To view a copy of this licence, visit http://creativecommons.org/licenses/by/4.0/.

\section{References}

Alves S, Mangas M, Prata MJ, Ribeiro G, Lopes L, Ribeiro H, PintoBasto J, Lima MR, Lacerda L (2006) Molecular characterization of Portuguese patients with mucopolysaccharidosis type II shows evidence that the IDS gene is prone to splicing mutations. 
J Inherit Metab Dis 29(6):743-754. https://doi.org/10.1007/ s10545-006-0403-z

Appelmans F, Wattiaux R, De Duve C (1955) Tissue fractionation studies. 5. The association of acid phosphatase with a special class of cytoplasmic granules in rat liver. Biochem J 3:438-445. https:// doi.org/10.1042/bj0590438

Appenzeller-Herzog C, Hauri HP (2006) The ER-Golgi intermediate compartment (ERGIC): in search of its identity and function. J Cell Sci 119(Pt 11):2173-2183. https://doi.org/10.1242/jcs.03019

Buratta S, Tancini B, Sagini K, Delo F, Chiaradia E, Urbanelli L, Emiliani C (2020) Lysosomal exocytosis, exosome release and secretory autophagy: the autophagic- and endo-lysosomal systems go extracellular. Int J Mol Sci 21(7):2576. https://doi.org/10.3390/ ijms21072576

Chen Y, Yu L (2017) Recent progress in autophagic lysosome reformation. Traffic 18(6):358-361. https://doi.org/10.1111/tra.12484

Chen Y, Yu L (2018) Development of research into autophagic lysosome reformation. Mol Cells 41(1):45-49. https://doi.org/10. 14348/molcells.2018.2265

De Araujo MEG, Liebscher G, Hess MW, Huber LA (2020) Lysosomal size matters. Traffic 21(1):60-75. https://doi.org/10.1111/ tra. 12714

Doherty GJ, McMahon HT (2009) Mechanisms of endocytosis. Annu Rev Biochem 78:857-902. https://doi.org/10.1146/annurev.bioch em.78.081307.110540

Eskelinen EL (2006) Roles of LAMP-1 and LAMP-2 in lysosome biogenesis and autophagy. Mol Aspects Med 27(5-6):495-502. https://doi.org/10.1016/j.mam.2006.08.005

Gaudet P, Livstone MS, Lewis SE, Thomas PD (2011) Phylogeneticbased propagation of functional annotations within the Gene Ontology consortium. Brief Bioinform 12(5):449-462. https:// doi.org/10.1093/bib/bbr042

Ghadially FN (1988) Lysosomes. In: Ghadially, FN (ed) Ultrastructural Pathology of the Cell and Matrix (Third Edition). ButterworthHeinemann, 589-765 https://doi.org/10.1016/B978-0-407-015722.50008-3

Go CD, Knight JDR, Rajasekharan A, Rathod B, Hesketh GG, Abe KT, Youn JY, Samavarchi-Tehrani P, Zhang H, Zhu LY, Popiel E, Lambert JP, Coyaud É, Cheung SWT, Rajendran D, Wong CJ, Antonicka H, Pelletier L, Palazzo AF, Shoubridge EA, Raught B, Gingras AC (2021) A proximity-dependent biotinylation map of a human cell. Nature 595(7865):120-124. https://doi.org/10.1038/ s41586-021-03592-2

Henne WM (2016) Organelle remodeling at membrane contact sites. J Struct Biol 196(1):15-19. https://doi.org/10.1016/j.jsb.2016.05. 003

Huotari J, Helenius A (2011) Endosome maturation. Embo J 30(17):3481-3500. https://doi.org/10.1038/emboj.2011.286

Huttlin EL, Ting L, Bruckner RJ, Gebreab F, Gygi MP, Szpyt J, Tam S, Zarraga G, Colby G, Baltier K, Dong R, Guarani V, Vaites LP, Ordureau A, Rad R, Erickson BK, Wühr M, Chick J, Zhai B, Kolippakkam D, Mintseris J, Obar RA, Harris T, ArtavanisTsakonas S, Sowa ME, De Camilli P, Paulo JA, Harper JW, Gygi SP (2015) The BioPlex network: a systematic exploration of the human interactome. Cell 162(2):425-440. https://doi.org/10. 1016/j.cell.2015.06.043

Jiang P, Nishimura T, Sakamaki Y, Itakura E, Hatta T, Natsume T, Mizushima N (2014) The HOPS complex mediates autophagosomelysosome fusion through interaction with syntaxin 17. Mol Biol Cell 25(8):1327-1337. https://doi.org/10.1091/mbc.E13-08-0447

Klionsky DJ, Eskelinen EL, Deretic V (2014) Autophagosomes, phagosomes, autolysosomes, phagolysosomes, autophagolysosomes... wait I'm confused. Autophagy. 10(4), 549-551.

Kobolák J, Molnár K, Varga E, Bock I, Jezsó B, Téglási A, Zhou S, Lo Giudice M, Hoogeveen-Westerveld M, Pijnappel WP, Phanthong P, Varga N, Kitiyanant N, Freude K, Nakanishi H, László L, Hyttel
P, Dinnyés A (2019) Modelling the neuropathology of lysosomal storage disorders through disease-specific human induced pluripotent stem cells. Exp Cell Res 380(2):216-233. https://doi.org/ 10.1016/j.yexcr.2019.04.021

Langemeyer L, Fröhlich F, Ungermann C (2018) Rab GTPase function in endosome and lysosome biogenesis. Trends Cell Biol 28(11):957-970. https://doi.org/10.1016/j.tcb.2018.06.007

Laszlo L, Lowe J, Self T, Kenward N, Landon M, McBride T, Farquhar C, McConnell I, Brown J, Hope J, Mayer RJ (1992) Lysosomes as key organelles in the pathogenesis of prion encephalopathies. J Pathol 166(4):333-341. https://doi.org/10.1002/path.1711660404

Lazarus GS, Hatcher VB, Levine N (1975) Lysosomes and the skin. J Invest Dermatol 65(3):259-271. https://doi.org/10.1111/15231747.ep12598332

Lemonnier T, Blanchard S, Toli D, Roy E, Bigou S, Froissart R, Rouvet I, Vitry S, Heard JM, Bohl D (2011) Modeling neuronal defects associated with a lysosomal disorder using patient-derived induced pluripotent stem cells. Hum Mol Genet 20(18):3653-3666. https://doi.org/10.1093/hmg/ddr285

Li S, Ghosh C, Xing Y, Sun Y (2020) Phosphatidylinositol 4,5-bisphosphate in the control of membrane trafficking. Int J Biol Sci 16(15):2761-2774. https://doi.org/10.7150/ijbs.49665

Liu S, Storrie B (2012) Are Rab proteins the link between Golgi organization and membrane trafficking? Cell Mol Life Sci 69(24):4093-4106. https://doi.org/10.1007/s00018-012-1021-6

Liu C, Mei M, Li Q, Roboti P, Pang Q, Ying Z, Gao F, Lowe M, Bao S (2017) Loss of the golgin GM130 causes Golgi disruption, Purkinje neuron loss, and ataxia in mice. Proc Natl Acad Sci USA 114(2):346-351. https://doi.org/10.1073/pnas.1608576114

Lőrincz P, Tóth S, Benkő P, Lakatos Z, Boda A, Glatz G, Zobel M, Bisi S, Hegedűs K, Takáts S, Scita G, Juhász G (2017) Rab2 promotes autophagic and endocytic lysosomal degradation. J Cell Biol 216(7):1937-1947. https://doi.org/10.1083/jcb.20161 1027

Lund VK, Madsen KL, Kjaerulff O (2018) Drosophila Rab2 controls endosome-lysosome fusion and LAMP delivery to late endosomes. Autophagy 14(9):1520-1542. https://doi.org/10.1080/ 15548627.2018.1458170

Luzio JP, Pryor PR, Bright NA (2007) Lysosomes: fusion and function. Nat Rev Mol Cell Biol 8(8):622-632. https://doi.org/10. 1038/nrm2217

Medina DL, Fraldi A, Bouche V, Annunziata F, Mansueto G, Spampanato C, Puri C, Pignata A, Martina JA, Sardiello M, Palmieri M, Polishchuk R, Puertollano R, Ballabio A (2011) Transcriptional activation of lysosomal exocytosis promotes cellular clearance. Dev Cell 21(3):421-430. https://doi.org/10.1016/j.devcel.2011. 07.016

Mellman I, Nelson WJ (2008) Coordinated protein sorting, targeting and distribution in polarized cells. Nat Rev Mol Cell Biol 9(11):833-845. https://doi.org/10.1038/nrm2525

Mizushima N, Yoshimori T (2007) How to interpret LC3 immunoblotting. Autophagy 3(6):542-545. https://doi.org/10.4161/auto.4600

Nixon RA, Cataldo AM (2006) Lysosomal system pathways: genes to neurodegeneration in Alzheimer's disease. J Alzheimers Dis 9(3 Suppl):277-289. https://doi.org/10.3233/jad-2006-9s331

Osaki Y, Saito A, Kanemoto S, Kaneko M (2018) Shutdown of ERassociated degradation pathway rescues functions of mutant iduronate 2-sulfatase linked to mucopolysaccharidosis type II. Cell Death Dis 9(8):808. https://doi.org/10.1038/s41419-018-0871-8

Palmieri M, Impey S, Kang H, di Ronza A, Pelz C, Sardiello M, Ballabio A (2011) Characterization of the CLEAR network reveals an integrated control of cellular clearance pathways. Hum Mol Genet 20(19):3852-3866. https://doi.org/10.1093/hmg/ddr306

Parkinson-Lawrence EJ, Shandala T, Prodoehl M, Plew R, Borlace GN, Brooks DA (2010) Lysosomal storage disease: revealing 
lysosomal function and physiology. Physiol (bethesda) 25(2):102115. https://doi.org/10.1152/physiol.00041.2009

Peter B, Lagzi I, Teraji S, Nakanishi H, Cervenak L, Zámbó D, Deák A, Molnár K, Truszka M, Szekacs I, Horvath R (2018) Interaction of positively charged gold nanoparticles with cancer cells monitored by an in situ label-free optical biosensor and transmission electron microscopy. ACS Appl Mater Interfaces 10(32):26841-26850. https://doi.org/10.1021/acsami.8b01546

Platt FM, Boland B, van der Spoel AC (2012) The cell biology of disease: lysosomal storage disorders: the cellular impact of lysosomal dysfunction. J Cell Biol 199(5):723-734. https://doi.org/10.1083/ jcb.201208152

Puertollano R (2014) mTOR and lysosome regulation. F Prime Rep. 6:52. https://doi.org/10.12703/p6-52

Rama Rao KV, Kielian T (2016) Astrocytes and lysosomal storage diseases. Neurosci 323:195-206. https://doi.org/10.1016/j.neuro science.2015.05.061

Rigon L, Kucharowski N, Eckardt F, Bauer R (2020) Modeling mucopolysaccharidosis type II in the fruit fly by using the RNA interference approach. Life (basel) 10(11):263. https://doi.org/10.3390/ life 10110263

Rigon L, De Filippis C, Napoli B, Tomanin R, Orso G (2021) Exploiting the potential of drosophila models in lysosomal storage disorders: pathological mechanisms and drug discovery. Biomedicines 9(3):268. https://doi.org/10.3390/biomedicines9030268

Rong Y, Liu M, Ma L, Du W, Zhang H, Tian Y, Cao Z, Li Y, Ren H, Zhang C, Li L, Chen S, Xi J, Yu L (2012) Clathrin and phosphatidylinositol-4,5-bisphosphate regulate autophagic lysosome reformation. Nat Cell Biol 14(9):924-934. https://doi.org/10. 1038/ncb2557

Samie MA, Xu H (2014) Lysosomal exocytosis and lipid storage disorders. J Lipid Res 55(6):995-1009. https://doi.org/10.1194/jlr. R046896

Scott CC, Vacca F, Gruenberg J (2014) Endosome maturation, transport and functions. Semin Cell Dev Biol 31:2-10. https://doi.org/ 10.1016/j.semcdb.2014.03.034

Spampanato C, Feeney E, Li L, Cardone M, Lim JA, Annunziata F, Zare H, Polishchuk R, Puertollano R, Parenti G, Ballabio A, Raben N (2013) Transcription factor EB (TFEB) is a new therapeutic target for Pompe disease. EMBO Mol Med 5(5):691-706. https://doi.org/10.1002/emmm.201202176

Tanida I, Ueno T, Kominami E (2004) LC3 conjugation system in mammalian autophagy. Int J Biochem Cell Biol 36(12):25032518. https://doi.org/10.1016/j.biocel.2004.05.009
Terman A, Brunk UT (1998) Lipofuscin: mechanisms of formation and increase with age. APMIS 106(2):265-276. https://doi.org/ 10.1111/j.1699-0463.1998.tb01346.x

Van de Beek J, Jonker C, Van de Welle R, Liv N, Klumperman J (2019) CORVET, CHEVI and HOPS - multisubunit tethers of the endolysosomal system in health and disease. J Cell Sci. https://doi.org/ $10.1242 /$ jcs. 189134

Varga E, Nemes C, Bock I, Varga N, Fehér A, Kobolák J, Dinnyés A (2006c) Generation of Mucopolysaccharidosis type II (MPS II) human induced pluripotent stem cell (iPSC) line from a 3-year-old male with pathogenic IDS mutation. Stem Cell Res 17(3):479 481. https://doi.org/10.1016/j.scr.2016.09.032

Varga E, Nemes C, Kovács E, Bock I, Varga N, Fehér A, Dinnyés A, Kobolák J (2016a) Generation of human induced pluripotent stem cell (iPSC) line from an unaffected female carrier of Mucopolysaccharidosis type II (MPS II) disorder. Stem Cell Res 17(3):514 516. https://doi.org/10.1016/j.scr.2016.09.035

Varga E, Nemes C, Bock I, Varga N, Fehér A, Dinnyés A, Kobolák J (2016b) Generation of Mucopolysaccharidosis type II (MPS II) human induced pluripotent stem cell (iPSC) line from a 1-year-old male with pathogenic IDS mutation. Stem Cell Res 17(3):482484. https://doi.org/10.1016/j.scr.2016.09.033

Varga E, Nemes C, Bock I, Varga N, Fehér A, Kobolák J, Dinnyés A (2016d) Generation of Mucopolysaccharidosis type II (MPS II) human induced pluripotent stem cell (iPSC) line from a 7-year-old male with pathogenic IDS mutation. Stem Cell Res 17(3):463465. https://doi.org/10.1016/j.scr.2016.09.034

Vitry S, Bruyère J, Hocquemiller M, Bigou S, Ausseil J, Colle MA, Prévost MC, Heard JM (2010) Storage vesicles in neurons are related to Golgi complex alterations in mucopolysaccharidosis IIIB. Am J Pathol 177(6):2984-2999. https://doi.org/10.2353/ ajpath.2010.100447

Wandinger-Ness A, Zerial M (2014) Rab proteins and the compartmentalization of the endosomal system. Cold Spring Harb Perspect Biol 6(11):a022616-a022616. https://doi.org/10.1101/cshpe rspect.a022616

Yip CK, Murata K, Walz T, Sabatini DM, Kang SA (2010) Structure of the human mTOR complex I and its implications for rapamycin inhibition. Mol Cell 38(5):768-774. https://doi.org/10.1016/j. molcel.2010.05.017 